\title{
Near-Infrared Light Emissions from Er-doped ZnO Thin Films Induced by an Electrical Field
}

\author{
Shigeru TANAKA, Yukari ISHIKAWA and Noriyoshi SHIBATA \\ Japan Fine Ceramics Center, 2-4-1, Mutsuno, Atsuta-ku, Nagoya-shi 456-8587
}

\begin{abstract}
We obtained near-infrared (NIR) emissions by applying an electric field to a $\mathrm{ZnO}$ thin film containing Er ions. The sample construction was indium tin oxide (ITO) film/Er-doped $\mathrm{ZnO}$ film/ITO film on a $\mathrm{SiO}_{2}$ glass substrate. By applying a suitable alternative voltage to the sample, a broad luminescence in the NIR wavelength range was observed. The intensity of the luminescence increased with applied voltage. The luminescence induced by the electric field had a wavelength of $1.5 \mu \mathrm{m}$, which suggests that the light emission is related to the intrashell transition of $\mathbf{E r}^{3+}$.

[Received February 21, 2007; Accepted April 19, 2007]
\end{abstract}

Key-words : Electroluminescence, ZnO, Thin film, Near-infrared emission, Erbium, Photoluminescence

Various types of optical/electronic devices are required in optical communication fields with a broad-band network system. Among the many types of material, zinc oxide $(\mathrm{ZnO})$ attracts considerable attention as one of the key materials because of its wide band gap of $3.3 \mathrm{eV}$ and large exciton binding energy of about $60 \mathrm{meV}$ at room temperature. Furthermore, $\mathrm{ZnO}$ shows the attractive characteristic that its electrical conductivity can be widely controlled from high conductivity to insulator by doping other elements. Recently, Tsukazaki et al. have succeeded in fabricating a stable p-type semiconductive $\mathrm{ZnO}$ thin film, which has led to the development of ultraviolet (UV) lasers and various types of light-emitting diodes (LEDs). ${ }^{1)}$ There has been much research on $\mathrm{ZnO}$ films, focusing on the UV emission mechanism and their nanostructures. On the other hand, little research has been reported on the photoluminescence $(\mathrm{PL})$ of $\mathrm{ZnO}$ thin films in the near-infrared (NIR) wavelength range. ${ }^{2)-4)}$ According to the results, an intense $1.54 \mu \mathrm{m}$ emission was obtained by doping Er to the $\mathrm{ZnO}$ matrix. The wavelength at $1.54 \mu \mathrm{m}$ corresponds to a low-loss wavelength of the conventional glass fiber; therefore, such a $\mathrm{ZnO}$ thin film has the potential of becoming useful devices in optical communications.

The electroluminescence (EL) technique using a fluorescent thin film has become wide spread for various types of display devices. ${ }^{5)}$ Although $\mathrm{ZnO}$ powder and $\mathrm{ZnO}$ composite phosphor thin films (i.e. $\mathrm{ZnGa}_{2} \mathrm{O}_{4}: \mathrm{Mn}$ ) have been reported, ${ }^{6}{ }^{-8}$ ) they are mainly restricted in their light emission characteristics to the visible wavelength range. To our knowledge, the EL of $\mathrm{ZnO}$-based thin films in the NIR wavelength range has not yet been reported. If the possibility of NIR emission from $\mathrm{ZnO}$ based thin films is verified, this will be important from the viewpoints of industrial and practical use, particularly for developing new devices.

On the basis of the above background, we demonstrated NIR emission from Er-doped $\mathrm{ZnO}$ thin films by applying an electrical field.

A simple construction was selected using a thin-film sample for EL investigation. $\mathrm{SiO}_{2}$ glass with a thickness of $0.5 \mathrm{~mm}$ was selected as a substrate. ITO thin films were used as the electrodes for applying a voltage to the fluorescent film. An ITO thin film was deposited on the substrate by reactive RF magnetron sputtering at $523 \mathrm{~K}$, and under a gas composition of $\mathrm{Ar} / \mathrm{O}_{2}(99 / 1)$. The Er-doped $\mathrm{ZnO}$ thin film having an area of $12 \times 15 \mathrm{~mm}^{2}$ was deposited on the ITO film. The Er-doped $\mathrm{ZnO}$ thin film was obtained by sputtering zinc metal and
$\mathrm{Er}_{2} \mathrm{O}_{3}$ targets simultaneously in an ambient gas mixture of $\mathrm{Ar} / \mathrm{O}_{2}(4 / 1)$ for $1 \mathrm{~h}$ at $623 \mathrm{~K}$. The concentration of $\mathrm{Er}$ in the $\mathrm{ZnO}$ matrix was estimated to be $\left.0.2 \mathrm{at} \%{ }^{4}\right)$ Then, the upper ITO film having an area of $8 \times 8 \mathrm{~mm}^{2}$ was deposited on the Er-doped $\mathrm{ZnO}$ film. A similar sample using undoped $\mathrm{ZnO}$ as a fluorescent thin film was also fabricated.

An AC electrical field $(13 \mathrm{~Hz})$ was applied to the Er-doped $\mathrm{ZnO}$ thin film, and light emission was measured using a $\mathrm{Ge}$ $p-i-n$ detector. PL measurements of the thin film sample were also carried out using the $351 \mathrm{~nm}$ of an $\mathrm{Ar}^{+}$laser. ${ }^{4)}$ The decay time of the specific emission at $1540 \mathrm{~nm}$ of EL was measured. X-ray diffraction (XRD) analysis and scanning electron microscopy (SEM) observation were performed to evaluate the structure of the thin-film sample. Infrared absorption measurements were carried out to confirm the IR transmittance of the ITO film.

Figure 1 shows (a) a photograph of the Er-doped $\mathrm{ZnO}$ thinfilm sample sandwiched between two ITO films and (b) the cross-sectional SEM image of the sample. The sample was almost transparent. The Er-doped $\mathrm{ZnO}$ film was about $1 \mu \mathrm{m}$ thick and the ITO films were about $200 \mathrm{~nm}$ thick. The XRD pattern of the sample confirmed that a $\mathrm{ZnO}(002)$ peak was predominant, although the fluorescent film was composed of poly crystals. This indicates that there were many columnar $\langle 001\rangle$-aligned grains in the Er-doped $\mathrm{ZnO}$ film synthesized under the experimental conditions. The undoped $\mathrm{ZnO}$ film sample showed results similar to those mentioned above. The transmittance of the ITO film in the NIR range was confirmed to be about $80 \%$.

Figure 2 shows the emission spectra in the NIR range from the Er-doped $\mathrm{ZnO}$ thin film induced by various electrical

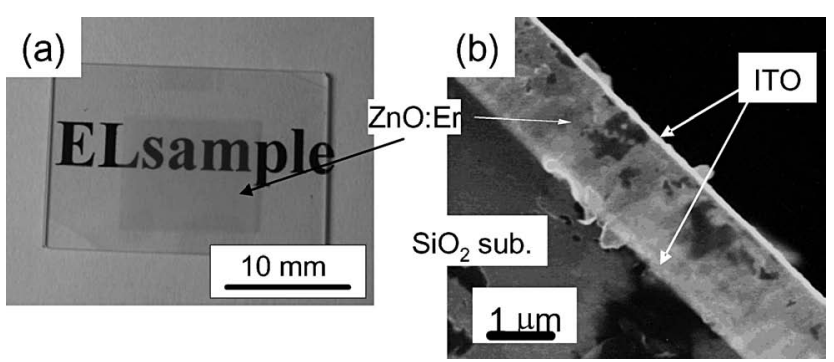

Fig. 1. Photographs of sample for EL measurement: (a) optical photograph, (b) cross-sectional SEM image. 


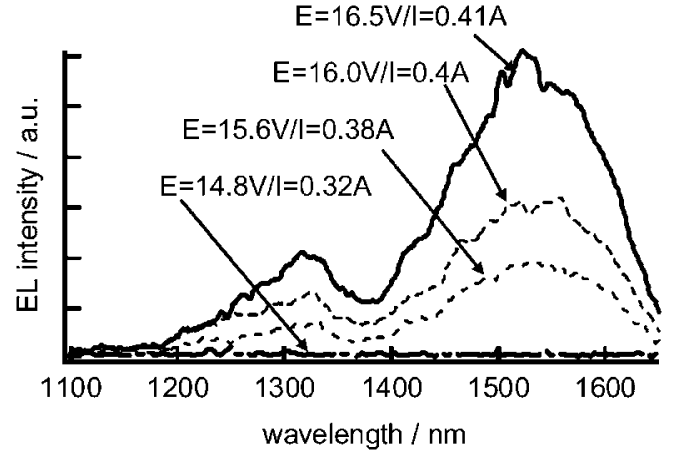

Fig. 2. EL spectra in NIR range from Er-doped $\mathrm{ZnO}$ thin film. The applied field for each spectrum is indicated.

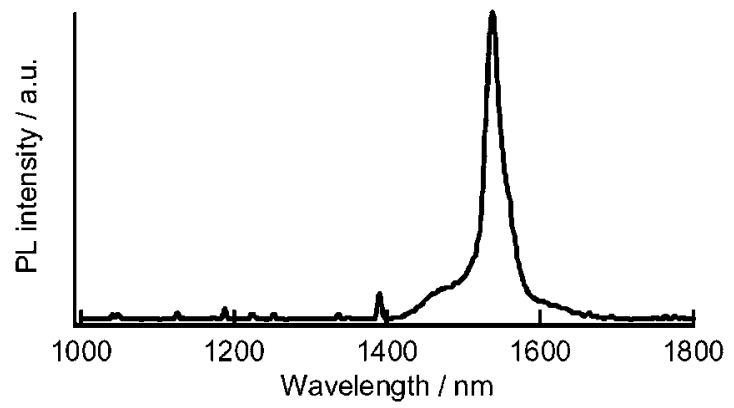

Fig. 4. Typical PL spectrum in NIR range from Er-doped $\mathrm{ZnO}$ thin film. Note that the spectral width is sharp compared with that of the EL spectra shown in Fig. 2. (a)

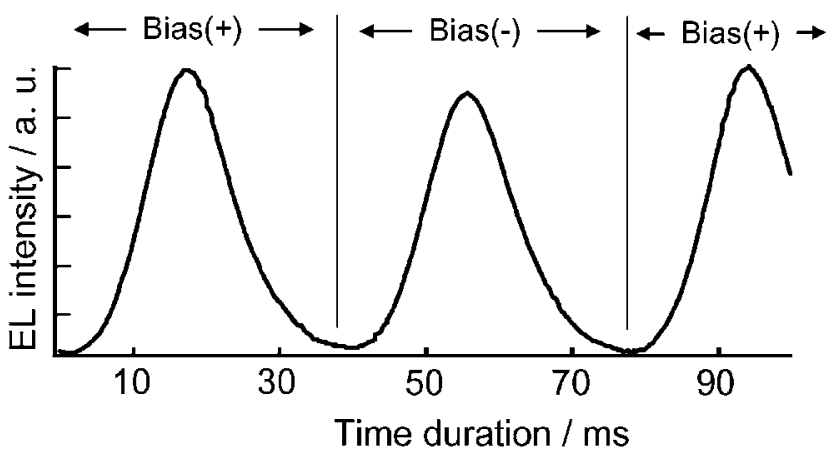

(b)

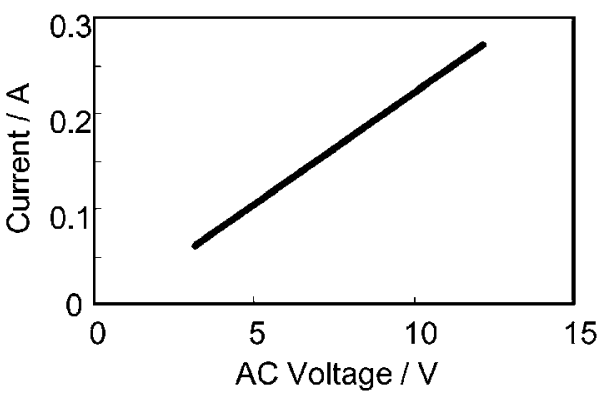

Fig. 3. (a) Emission pattern at $1540 \mathrm{~nm}$ under AC bias and (b) typical voltage-current characteristics (AC) of Er-doped $\mathrm{ZnO}$ thin film.

fields. Broad emission was observed at $\pm 15.6 \mathrm{~V}$. With a gradual increase in the applied voltage, the emission intensity increased. The maximum intensity of the emission was obtained at $\mathrm{V}= \pm 16.5 \mathrm{~V}$. When the applied voltage was lower than $14.8 \mathrm{~V}$, luminescent peaks could hardly be observed within the detection sensitivity of the apparatus. On the other hand, there was no emission signal from the Er-doped $\mathrm{ZnO}$ sample in another range from $370 \mathrm{~nm}$ to $1000 \mathrm{~nm}$ under the conditions described above $(V=16.5 \mathrm{~V})$. In the case of the undoped $\mathrm{ZnO}$ thin film, luminescence was not detectable in the entire range: $370 \mathrm{~nm}-1800 \mathrm{~nm}$ under a similar electrical field $(V=26.5 \mathrm{~V})$.

Figure 3 shows (a) a typical oscillograph of the emission intensity at $1540 \mathrm{~nm}$ in the direction of applied voltage and (b) the voltage-current characteristics (AC) of the sample. It was found that the EL intensity was independent of the direction of the bias and that the $V-I$ characteristics of the sample were almost linear, this being an intrinsic property of $\mathrm{ZnO}$. These results indicate that the emission obtained in this experiment did not originate from electron-hole recombination in a pn junction or a Schottky barrier but a direct or indirect excitation/transition of ions induced by electrically accelerating electrons.

The decay time of the emission at $1540 \mathrm{~nm}$ was measured by applying a short pulse to the sample, and its value was about $2.5 \mathrm{~ms}$, which was in agreement with the reported value for the PL peak in Er-doped $\mathrm{ZnO}$ thin film. ${ }^{3)}$ In addition, the luminescence shown in Fig. 3(a) disappeared immediately when the applied voltage was removed. Taking account of these phenomena and the lack of emission from the undoped
$\mathrm{ZnO}$ sample, it is thought to be that the emission is not obtained owing to a thermal effect but to an electrical effect and is strongly related to the intrashell transition of $\mathrm{Er}^{3+}$ $\left({ }^{4} I_{13 / 2} \rightarrow{ }^{4} I_{15 / 2}\right)$.

Figure 4 shows a typical PL spectrum in the NIR range from the Er-doped $\mathrm{ZnO}$ thin film. A sharp spectral peak having a center wavelength at $1540 \mathrm{~nm}$ was observed in contrast to the EL spectrum shown in Fig. 2. It is difficult to interpret the detail of the EL spectrum of Fig. 2; for example, the reason why there are broad spectral shapes compared to the PL spectrum and the identification of the peaks at approximately 1.3 $\mu \mathrm{m}$ are still unresolved. This means such a specific EL spectral shape in the NIR region is thought to suggest that the energy states of Er ions in the $\mathrm{ZnO}$ matrix (or $\mathrm{ZnO}$-Er atomic interaction) are complex under a high electrical field.

By applying a suitable alternating electrical field to Erdoped $\mathrm{ZnO}$ thin films, a broad luminescence in the NIR wavelength range was observed. The luminescence showed high emission intensity in the vicinity of the wavelength of 1.5 $\mu \mathrm{m}$, which suggests that the light emission is related to the presence of Er ions.

Acknowledgements This study was partly supported under the auspices of the Special Coordination Fund "Ceramic Integration Project" from METX, The Murata Scientific Foundation and Iketani Science Technology Foundation and Research for Promoting Technological Seeds from JST. 


\section{References}

1) Tsukazaki, A., Ohtomo, A., Onuma, T., Ohtani, M., Makino, T., Sumiya, M., Ohtani, K., Chichibu, S. F., Fuke, S., Segawa, Y., Ohno, H., Koinuma, H. and Kawasaki, M., Nature Materials, Vol. 4, pp. 42-46 (2005).

2) Casero, R. P., Llorente, A. G.., Y. Moll, O. P., Seiler, W., Defoumeau, R. M., Defoumeau, D., Millon, E., Perriere, J., Goldner, P. and Viana, B., J. Appl. Phys., Vol. 97, pp. 054905-1-8 (2005).

3) Komuro, S., Katsumata, T., Morikawa, T., Zhao, X., Isshiki, H. and Aoyagi, Y., J. Appl. Phys., Vol. 88, pp. 7129-7136 (2000).
4) Ishikawa, Y., Okamoto, M., Tanaka, S., Nezaki, D. and Shibata, N., J. Mater. Res., Vol. 20, pp. 2578-2583 (2005).

5) Yoshida, M., Mikami, A. and Inoguchi, T., "Phosphor Handbook," Ed. by Shionoya, S. and Yen, W. M., CRC press, U.S.A. (1999) Chap. 3, pp. 581-612.

6) Minami, T., Maeno, T., Kuroi, Y. and Takata, S., Jpn. J. Appl. Phys., Vol. 34, pp. L684-L687 (1995).

7) Minami, T., Miyata, T., Takata, S. and Fukuda, I., Jpn. J. Appl. Phys., Vol. 30, pp. L117-L119 (1991).

8) Bhushan, S., Pandey, A. N. and Kaza, B. R., J. Luminescence, Vol. 20, pp. 29-38 (1979). 